\title{
HOT DISKS AND DELAYED BAR FORMATION
}

\author{
Kartik Sheth $^{1}$, Jason Melbourne ${ }^{2}$, Debra Meloy Elmegreen ${ }^{3}$, Bruce G. Elmegreen ${ }^{4}$, E. Athanassoula ${ }^{5}$, \\ Roberto G. ABraham ${ }^{6}$, AND BenJAMin J. WeINER ${ }^{7}$ \\ ${ }^{1}$ National Radio Astronomy Observatory, 520 Edgemont Road, Charlottesville, VA 22903, USA \\ ${ }^{2}$ Department of Astronomy California Institute of Technology, MC 105-24, 1200 East California Boulevard, Pasadena, CA 91125, USA \\ ${ }^{3}$ Department of Physics and Astronomy, Vassar College, 124 Raymond Avenue, Poughkeepsie, NY 12604, USA \\ ${ }^{4}$ IBM T. J. Watson Center, P.O. Box 218, Yorktown Heights, NY 10598, USA \\ ${ }^{5}$ LAM (Laboratoire d'Astrophysique de Marseille), Aix Marseille Universite, CNRS, UMR 7326, F-13388 Marseille, France \\ ${ }^{6}$ Canadian Institute for Theoretical Astrophysics, Mclennan Labs, University of Toronto, 60 St. George St, Room 1403, Toronto, ON M5S 3H8, Canada \\ ${ }^{7}$ Department of Astronomy/Steward Observatory, 933 North Cherry Avenue, Tucson, AZ 85721-0065, USA \\ Received 2012 July 30; accepted 2012 August 30; published 2012 October 9
}

\begin{abstract}
We present observational evidence for the inhibition of bar formation in dispersion-dominated (dynamically hot) galaxies by studying the relationship between galactic structure and host galaxy kinematics in a sample of 257 galaxies between $0.1<z \leqslant 0.84$ from the All-Wavelength Extended Groth Strip International Survey and the Deep Extragalactic Evolutionary Probe 2 survey. We find that bars are preferentially found in galaxies that are massive and dynamically cold (rotation-dominated) and on the stellar Tully-Fisher relationship, as is the case for barred spirals in the local universe. The data provide at least one explanation for the steep $(\times 3)$ decline in the overall bar fraction from $z=0$ to $z=0.84$ in $L^{*}$ and brighter disks seen in previous studies. The decline in the bar fraction at high redshift is almost exclusively in the lower mass $\left(10<\log M_{*}\left(M_{\odot}\right)<11\right)$, later-type, and bluer galaxies. A proposed explanation for this "downsizing" of the bar formation/stellar structure formation is that the lower mass galaxies may not form bars because they could be dynamically hotter than more massive systems from the increased turbulence of accreting gas, elevated star formation, and/or increased interaction/merger rate at higher redshifts. The evidence presented here provides observational support for this hypothesis. However, the data also show that not every disk galaxy that is massive and cold has a stellar bar, suggesting that mass and dynamic coldness of a disk are necessary but not sufficient conditions for bar formation-a secondary process, perhaps the interaction history between the dark matter halo and the baryonic matter, may play an important role in bar formation.
\end{abstract}

Key words: galaxies: evolution - galaxies: high-redshift - galaxies: spiral - galaxies: structure

\section{BACKGROUND}

The presence of galactic structures such as bars is an important signpost in the evolution of a galaxy disk. Analytical work and simulations have shown that, once a galaxy disk is sufficiently massive and dynamically cold, the formation of a stellar bar is relatively fast ( $\sim$ hundred million years) (e.g., Hohl 1971; Kalnajs 1972; Ostriker \& Peebles 1973; Sellwood \& Wilkinson 1993; Athanassoula 2002, 2003; Heller et al. 2007). But bar formation can be delayed either by an initially dominant dark matter (DM) halo and/or a dynamically hot/dispersion-dominated disk $^{8}$ (Athanassoula \& Sellwood 1986)

An initially dominant DM halo strongly impacts the timescale for bar formation, delaying the onset of the bar instability (Athanassoula 2002). The bar that ultimately forms in such a system is stronger than a bar that would form in an otherwise non-DM-dominated galaxy because the DM halo acts as an efficient sink of angular momentum and energy for baryons, which are redistributed to form the bar. As the bar grows it pushes material inward so that the baryonic matter can become the dominant mass component in the inner parts of galaxies (Athanassoula \& Misiriotis 2002). Simulations also show that a dynamically hot disk delays bar formation (Athanassoula \& Sellwood 1986; Athanassoula 2003) because when random motions of stars in a disk have a higher amplitude than rotational ordered motions, the bar instability cannot grow quickly. This

\footnotetext{
8 We use the terms dynamically hot and dispersion-dominated interchangeably throughout the paper.
}

may even push the bar formation timescale beyond a Hubble time.

A recent COSMOS study of over $2000 L^{*}$ and brighter, faceon $\left(i<65^{\circ}\right)$ disk galaxies showed that the overall bar fraction $\left(f_{\text {bar }}=\right.$ total number of barred galaxies divided by the total number of disk galaxies) in disk galaxies declines sharply from $f_{\text {bar }} \sim 0.65$ at $z=0$ to $f_{\text {bar }}<0.2$ at $z=0.84$ (Sheth et al. 2008). It is crucial to note that the COSMOS sample is a complete sample only for disks with stellar masses $M_{*}>10^{10} M_{\odot}$; the published results of the bar fraction evolution apply only to this mass range (Sheth et al. 2008; Cameron et al. 2010). Therefore, studies with samples of lower mass galaxies $\left(M_{*}<10^{10} M_{\odot}\right.$, such as those typically done for nearby galaxies; e.g., using Sloan Digital Sky Survey) are not directly comparable to high-redshift studies.

The evolution of the bar fraction with redshift is not uniform across all disk galaxies. As a function of redshift, $f_{\text {bar }}$ is strongly correlated with the host galaxy mass, color, and bulge dominance (see Figures 2-5 in Sheth et al. 2008). The most massive stellar disks $\left(M_{*} \geqslant 10^{11} M_{\odot}\right)$, which are also redder and have a larger bulge, already had $f_{\text {bar }}>0.5$ at $z \sim 0.8$, nearly the present-day value of their bar fraction. In sharp contrast, the lower stellar mass systems $\left(M \sim 10^{10} M_{\odot}\right)$ had $f_{\text {bar }} \ll 0.2$ at $z \sim 0.8$. Over the last $7 \mathrm{Gyr}$, the lower mass galaxies have evolved the fastest, increasing their bar fraction by more than a factor of three, to their present-day value of $f_{\text {bar }} \sim 0.65$. This behavior is another form of "downsizing" (Cowie et al. 1996).

The dynamics of high-redshift disks has been a hot topic of study in recent years (e.g., Kassin et al. 2007; Förster Schreiber et al. 2009; Cresci et al. 2009; Fernández Lorenzo et al. 2009; 
Davies et al. 2011; Miller et al. 2011; Kassin et al. 2012). At high redshifts $(z \geqslant 2)$, there is evidence for both rotationand dispersion-dominated disks (e.g., Law et al. 2009; Cresci et al. 2009; Wright et al. 2009), although the evolution of the disk kinematics and assembly is not well understood. The dynamics of a galaxy must change as it acquires mass, undergoes interactions/mergers, and forms stars. In this paper, we seek to understand how the disk dynamics are influencing the formation of bars.

In a 2007 study of over $\sim 500$ galaxies from $0.2<z<$ 1.2, Kassin et al. (2007) found that major mergers, disturbed and compact systems are preferentially off the stellar mass Tully-Fisher (TF) relationship toward lower rotational velocities. In contrast, for the local universe, Barton et al. (2001), who examined 90 close pairs, found that only eight scattered off the TF relationship. Kannappan et al. (2002) analyzed the residuals in the TF for a wide variety of galaxy morphologies and environments from the Nearby Field Galaxy Survey and found that the scatter in the TF did not change once corrections for dust extinction and star formation were applied, although the scatter did increase for non-spiral galaxies. They also found that dwarf galaxies did not follow the TF with dwarfs scattering on both sides of the TF (see Kannappan et al. 2002 for an in-depth discussion). At high redshifts, Kassin et al. (Figure 1, 2007) suggest that more early-type spirals (blue squares in their Figure 1) are on the classical stellar TF relationship compared to late-type/irregular spirals. It appears that over time, more and more of the late-type/irregular galaxies arrive onto the stellar TF. A different study of disk-like galaxies by Miller et al. (2011) has argued that there is no significant evolution in the stellar mass TF relationship to $z \sim 1$, although there is an evolutionary trend in the $B$-band TF. While the precise evolution of the stellar TF is not known, we make use of the existing measurements of disk properties (mass, rotational velocity, and velocity dispersion) from Kassin et al. (2007) and compare these for different types of galaxies.

\section{DEFINING THE GALAXY SAMPLE FOR CLASSIFICATION OF GALACTIC STRUCTURE}

We began with the 544 emission-line galaxies studied by Kassin et al. (2007). For each of these galaxies, we made a cutout of the $V$ - and $I$-band Advanced Camera for Surveys (ACS) images and visually examined each fits file. Disk galaxies inclined more than $65^{\circ}$, as measured by Kassin et al. (2007), were discarded. Note that an inclination cut was already made by Kassin et al. (2007), removing galaxies with $i<30^{\circ}$ and $i>70^{\circ}$. We also removed obviously merging galaxies and restricted the sample to $z<0.84$, following our detailed analysis of the band-shifting effect on identification of bars in Sheth et al. (2008). Beyond this redshift, the rest-frame wavelength for ACS I-band images shifts shortward of the $4000 \AA$ break where bar identification becomes difficult (see Figures 7, 8, and 13 in Sheth et al. 2008). We also eliminated any galaxy fainter than $L_{V}^{*}$ with an empirically determined luminosity evolution of $1 \mathrm{mag}$ from Capak (2003) such that $M_{V}^{*}=-21.7$ at $z=0.9$ (Capak 2003). These criteria are based on the detailed analysis of selection effects and sample selection which are critical for high-redshift studies as discussed in detail in Sheth et al. (2008).

Each of the galaxies were classified independently by four authors (D.M.E., B.G.E., K.S., and J.M.) into the following classifications: barred, unbarred, clumpy (or clump-cluster), chain, and compact galaxies using postage stamps made from the optical Hubble Space Telescope data from the All-Wavelength
Extended Groth Strip International Survey (AEGIS; Davis et al. 2007). The criteria followed were as follows: a barred galaxy was one that showed an obvious recognizable bar. The bars were further divided into "long" and "short" bars_- "long" bars were those that subtended more than half of the galaxy disk. The chain and clumpy galaxies were identified following the previous work by, e.g., Elmegreen et al. (2004, 2005, 2007)—these are nascent galaxies with several bright star-forming clumps believed to be in their first epoch of fragmentation and star formation. Chain galaxies are believed to be clumpy galaxies viewed edge-on. The agreement between the authors was excellent with only a $4 \%$ disagreement between the barred versus unbarred cases. This $4 \%$ sample was then jointly debated and analyzed and a reconciled classification was made between all the authors. Examples of each classification class are shown in Figure 1. The final sample has 126 unbarred disk galaxies, 28 long bars, 20 short bars, 22 clumpy, 12 chain, and 49 compact galaxies, for a total of 257 galaxies. The sample is not large enough to be statistically complete or robust, as was the case for the COSMOS sample, but it is sufficient to show the basic relationship between the galaxy host kinematics and the development of galactic structures. We also studied the galaxies as a function of redshift, but the number of galaxies per redshift bin of $\delta z=0.1$ was then so small $(\leqslant 50)$ that when divided further by galaxy type, the results were not statistically meaningful.

\section{GALAXY KINEMATICS}

The kinematics of the galaxies in this sample were measured from Keck DEIMOS spectra obtained by the Deep Extragalactic Evolutionary Probe 2 (DEEP2) Survey (Davis et al. 2003, 2007; Newman et al. 2012) with the 1200 line $\mathrm{mm}^{-1}$ grating. The kinematic measurements were first presented in Kassin et al. (2007). As the details of the observations and measurement techniques have been described elsewhere (Weiner et al. 2006a, 2006b; Kassin et al. 2007), we simply summarize the key points here: a measure of the velocity dispersion and rotation was made from multiple bright emission lines (typically $\mathrm{H} \beta$, [O II] $\lambda 3727$, and [O III] $\lambda$ 5007). These quantities were measured directly from the two-dimensional spectral images with a routine called ROTCURVE (Weiner et al. 2006a). ROTCURVE constructs models of the two-dimensional emission-line structure, convolves those models with the atmospheric seeing $(\sim 0$ !'7), compares them to the data, and then provides a chi-squared best fit to the values of line intensity, velocity dispersion, and rotation. Rotational velocities, if present, were detectable down to $\sim 5 \mathrm{~km} \mathrm{~s}^{-1}$, whereas dispersions were resolvable to $\sim 15 \mathrm{~km} \mathrm{~s}^{-1}$ (a limit set by the spectral resolution of $\sim 25 \mathrm{~km} \mathrm{~s}^{-1}$ ). For galaxies with physical sizes smaller than the seeing, the ROTCURVE fit is a lower limit to the rotation velocity and an upper limit to the dispersion.

In Figure 2, we examine the individual measurements of the dispersion and rotational velocities as a function of galaxy type-about one quarter of the compact galaxies have a rotation velocity measurement that is determined to be lower than the nominal $5 \mathrm{~km} \mathrm{~s}^{-1}$ limit for these data and should be treated with caution. Similarly about $\sim 10 \%$ of the unbarred disk galaxies and a handful of short bars and compact systems have a measured velocity dispersion below $15 \mathrm{~km} \mathrm{~s}^{-1}$. These systems are marked with a black circle and should be considered uncertain.

An important caveat to note about our use of velocity dispersions from emission-line measurements is that these may be very different from the stellar velocity dispersion because the measurements primarily reflect the mean of the local 

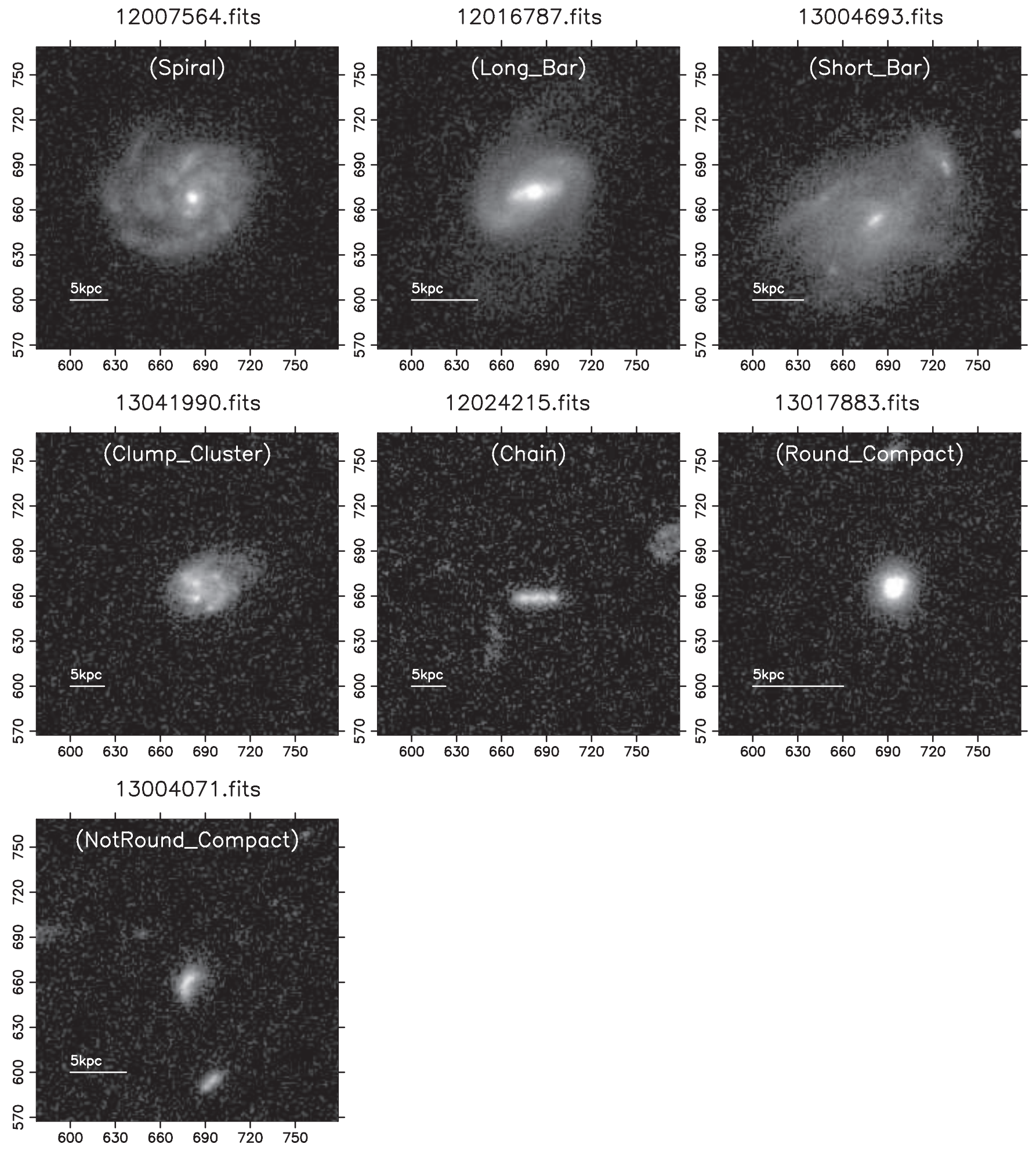

Figure 1. Example for each of the classification class used to identify the galaxies from the parent DEEP2/AEGIS sample as described in Section 2. Each panel shows the cutout of a type of galaxy. The redshift and stellar mass are indicated at the top, the classification class is shown at the bottom with the total number in each class indicated in the parenthesis. Also shown is a line segment for $5 \mathrm{kpc}$ at that redshift and a line segment to indicate 20 pixels—-the compact galaxies are usually smaller than these segments.

gas velocity dispersion from star-forming regions. In nearby galaxies the velocity dispersion in $\mathrm{H}$ II regions can easily reach several tens of $\mathrm{km} \mathrm{s}^{-1}$ and the velocity dispersion from emission lines may over- or underestimate the stellar velocity dispersion (see also Davies et al. 2011). This important caveat, however, does not affect the main analysis presented here, which relies on the structure of galaxies in comparison to their deviation from the TF relation.

\section{RESULTS}

We combine the data from the Kassin et al. (2007) study with our classifications described above. The results are plotted 


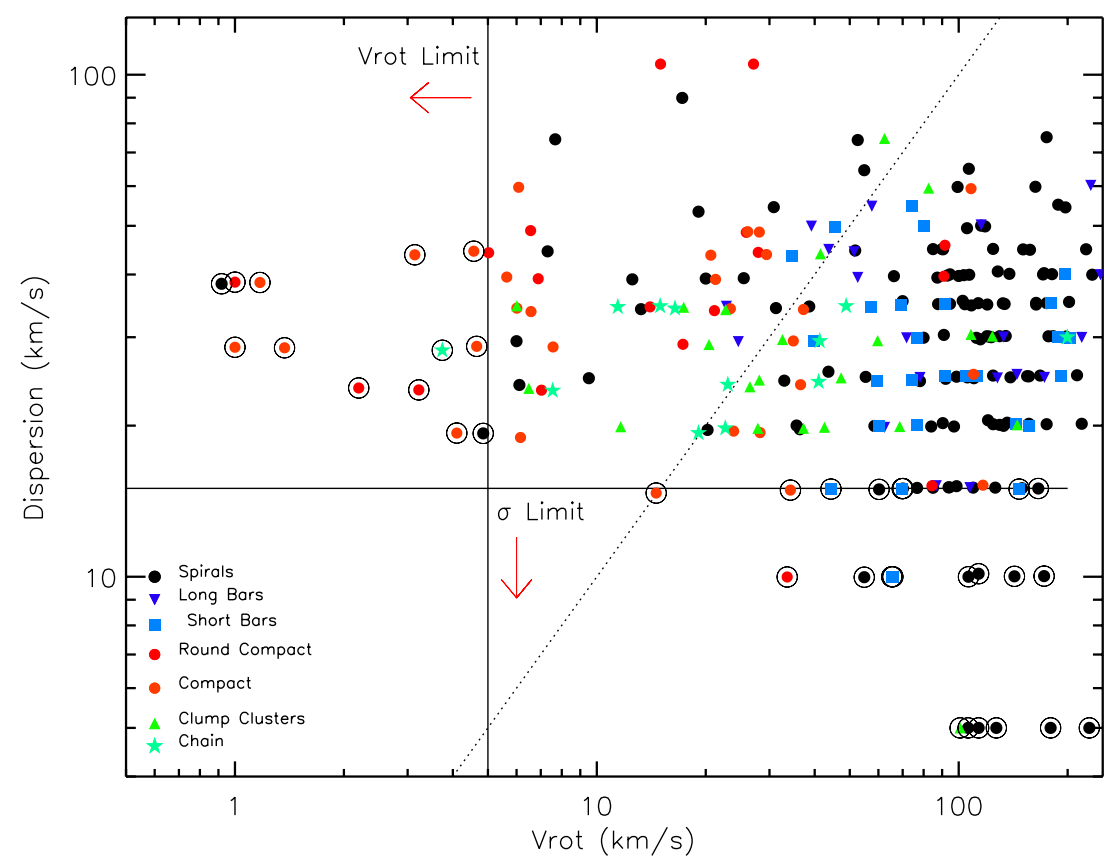

Figure 2. Plot of the measured velocity dispersion vs. inclination-corrected rotational velocity for different types of galaxies. The solid black lines with red arrows indicate the limits of the measurements. The diagonal dotted line is where the velocity dispersion and rotation velocities are equal. Galaxies that fall below the nominal limits for these data are encircled with a black circle and are marked as such in all subsequent figures.

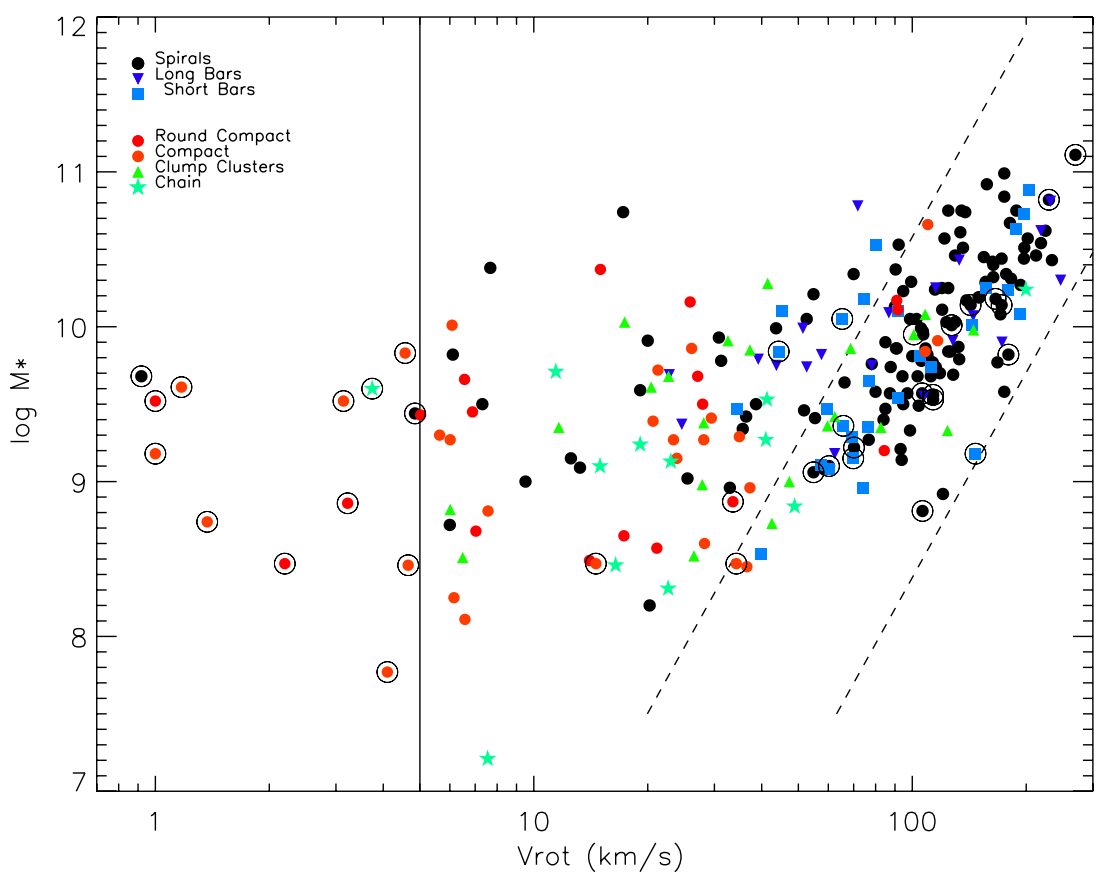

Figure 3. Stellar mass and rotational velocities for the different galaxy-type classifications are plotted. The dashed lines show the width of the stellar TF relationship between $z \sim 0$ to $z \sim 1$ as derived by Bell \& de Jong (2001) and Conselice et al. (2005), respectively (and as shown in Figure 1 of Kassin et al. 2007). The vertical solid line is the same as that in Figure 2, showing the limits of our measurement. The symbols for the different galaxies are as follows: dark filled circles, unbarred disks; blue triangles, long bars; light blue rectangles, short bars; filled red circles, round compact; filled orange circles, non-round compact; green triangles; clump clusters, and filled stars are chain galaxies. The black circles encircling some of the data points indicate galaxies for which the measured rotational velocity or velocity dispersion are uncertain due to the limitations of the observations.

in Figures 3 and 4. These two figures show the main result of this study-barred spirals (blue squares and purple triangles) in this high-redshift sample are primarily found in massive, rotationally supported galaxies that are on the $\mathrm{TF}$, as is the case for galaxies in the local universe (Courteau et al. 2003). There is a large "tail" of galaxies toward the lower rotational velocities primarily made of compact (red or orange circles) and clumpy/chain (green triangles/stars) systems. There are a few $(\sim 20 / 126$ or $15 \%)$ unbarred disk galaxies in the region off the TF but there are virtually no barred spirals far from the envelope of the TF.

In Figure 4, we plot the TF shown in Figure 3 but now colorcoded with "dynamic hotness," i.e., using the ratio of the velocity dispersion to the rotational velocity $\left(h=\sigma / V_{\text {rot }}\right)$ and separated by galaxy type in each of the six panels. Galaxies with $\log h>$ 0 are dispersion-dominated, whereas those with $\log h<0$ are 

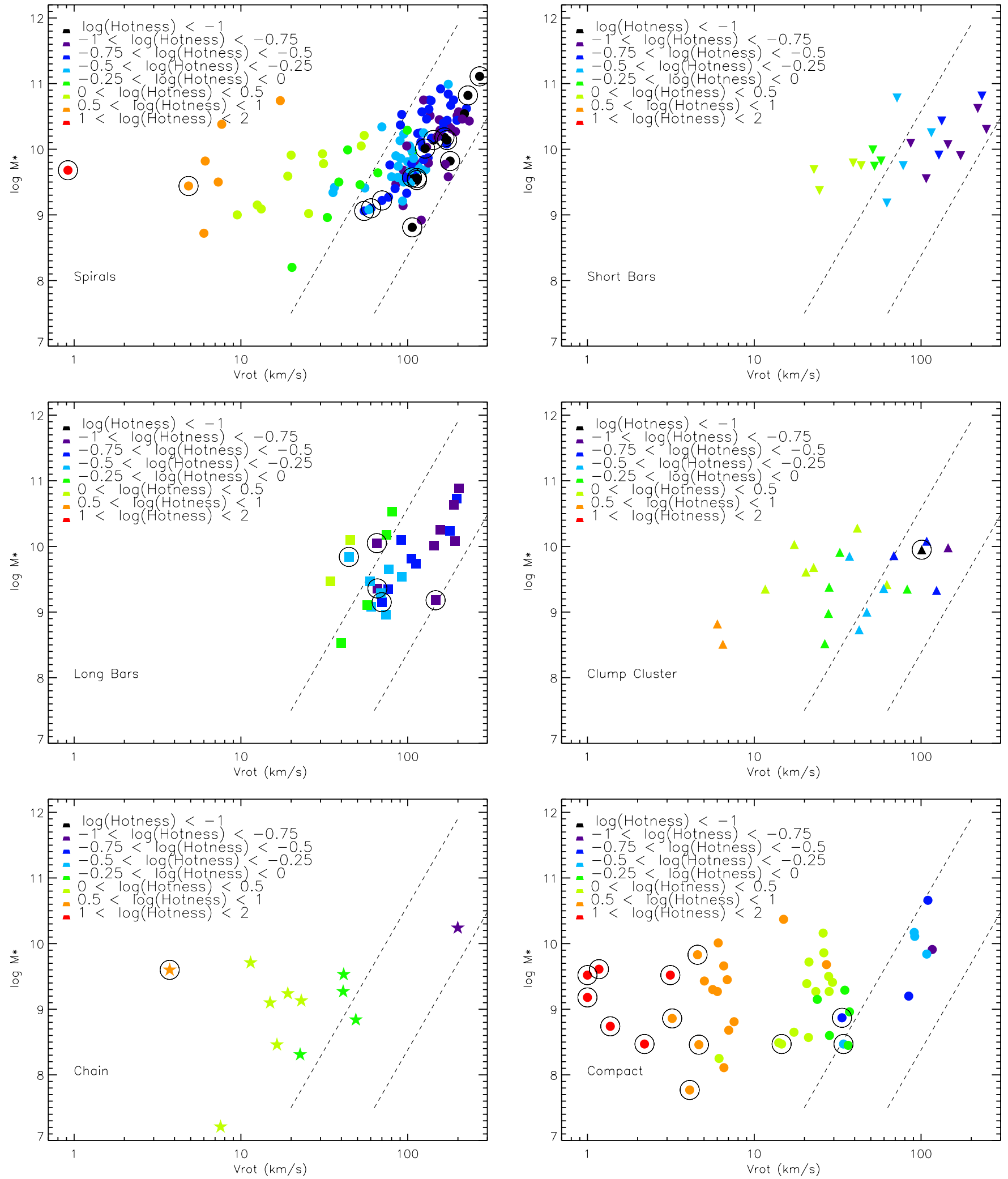

Figure 4. Symbols are color-coded based on the dynamical hotness of the galaxy. The hotness bins are $\log h<-1$ (black), $-1<\log h<-0.75$ (purple), $-0.75<$ $\log h<-0.5$ (dark blue), $-0.5<\log h<-0.25$ (light blue), $-0.25<\log h<0$ (dark green), $0<\log h<0.5$ (light green), $0.5<\log h<1$ (orange), and $1<\log h<$ 2 (red). The location of the galaxies on the stellar TF is strongly correlated with the hotness of the disk. There is a gradual progression from dispersion-dominated systems to rotationally dominated systems from the left to right in every type of galaxy, including within the barred galaxies.

rotation-dominated. As noted by Kassin et al. (2007), there is a clear trend with galaxies migrating to the $\mathrm{TF}$ with decreasing hotness. As would be expected, the rotation-dominated systems (blue-black colors) are on the TF and are primarily made of unbarred and barred spirals. About half of the clumpy galaxies and a few compact systems are also rotation-dominated, whereas the dispersion-dominated systems are predominantly composed of compact galaxies. One difference to note is the dynamic state 


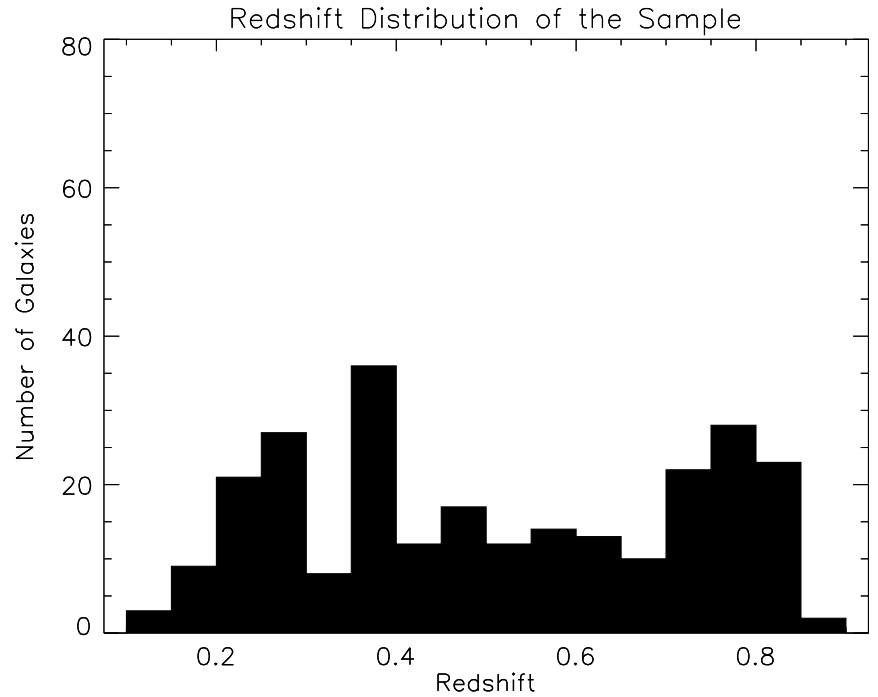

Figure 5. Redshift distribution for the 257 galaxies in this sample is shown here. The median redshift of the sample is 0.46 . There are too few galaxies in each redshift bin to discuss evolutionary trends with both mass and redshift, which will have to wait for even larger surveys.

of the barred population- $20 \%$ of the long bars are coded light or dark green, indicating $-0.25<\log h<0.5$, compared to $40 \%$ of the short bars. Although the sample sizes are relatively small, the difference suggests that short bars are preferentially in somewhat hotter disks than long bars.

\section{DISCUSSION}

The main result of this paper is that bars are not present in dispersion-dominated disk galaxies. The data suggest an evolutionary sequence in the assembly of disks and formation of the familiar galactic structures, such as bars, that we see today. The clump-cluster and chain galaxies are believed to be an early phase of present-day spiral galaxies undergoing a burst of star formation in large, gravitationally unstable clumps in a cold, gaseous disk (e.g., Elmegreen et al. 2005, 2009). As these disks evolve and accrete more cold material from the large-scale structure filaments, they should evolve toward more rotationally supported disks, the type that we see on the TF relationship (see also the discussion in Kassin et al. 2007). While it is not clear how these systems migrate from the left-hand side of the diagram to the right, the expected evolution is likely to be toward the higher rotational velocities and stellar masses (up and to the right) in Figure 3. The data also suggest that bars may be growing from short to long as the disks evolve into colder and more massive systems, indicating that bar-driven heating of the disk is less significant than the competing cooling processes.

Previous studies have shown a strong correlation between the bar fraction, stellar mass of the galaxy, and redshift such that massive galaxies $\left(>10^{11} M_{\odot}\right)$ had a high $(>50 \%)$ bar fraction at $z \sim 0.85$, whereas lower mass galaxies $\left(10^{10} M_{\odot}\right)$ had a bar fraction $<20 \%$ (Sheth et al. 2008). The evolution of the bar fraction was differential over the last $7 \mathrm{Gyr}$, with the fastest growth of the bar fraction occurring in the low-mass, blue, late-type spirals of masses between $10^{10}$ and $10^{11} M_{\odot}$ (Sheth et al. 2008; Cameron et al. 2010). The present DEEP2/AEGIS sample is too small to measure the redshift and mass-dependent evolution of these galaxies. The redshift distribution is shown in Figure 5. While we do see a segregation along the stellar mass axis between compact galaxies and disk (barred and unbarred) galaxies there are too few galaxies to infer any trends with mass and redshift - further analysis with larger data sets will be very useful in interpreting the evolutionary trends with mass and redshift. Finally, the compact systems, which are primarily dispersion-dominated, are seen over the entire redshift range of this survey and are therefore not necessarily only exotic highredshift systems, as was found in Kassin et al. (2007). The fate of these objects is another interesting area of study, especially at lower redshifts, where high spatial resolution (and higher signal-to-noise) is available.

Although our data shed some light on the conditions that delay bar formation, the large number of non-barred galaxies that are massive, cold, and rotationally supported remains a mystery. Courteau et al. (2003) have already shown that there is no obvious difference in the placement of barred and unbarred spirals on the TF in the local universe. Locally, as many as $30 \%-35 \%$ of the disk galaxies are unbarred. And so we conclude that while dynamic coldness and sufficient stellar mass are necessary conditions for the formation of a bar, they are not sufficient. Mergers and interactions are other processes that could play a role in bar formation but their impact is difficult to quantify because they can create long-lived or transient bars or they can destroy existing bars (Gerin et al. 1990; Barnes \& Hernquist 1991; Mihos \& Hernquist 1994, 1996; RomanoDíaz et al. 2008)). Finally, another important process for bar formation is the interaction history between the baryonic matter and the DM halo, especially in the inner parts of galaxies because the DM halo can act as a sink of angular momentum and energy for the baryonic matter settling into the central bar (e.g., Athanassoula 2002).

We thank the referee for the careful reading and suggestions that improved the paper. We are also grateful to the DEEP2 team for graciously sharing their data for this analysis. We thank Richard Ellis, Rick Fisher, Sarah Miller, and Preethi Nair for insightful discussions that helped improve this paper. K.S. acknowledges support from the National Radio Astronomy Observatory is a facility of the National Science Foundation operated under cooperative agreement by Associated Universities, Inc. E.A. acknowledges financial support to the DAGAL network from the People Programme (Marie Curie Actions) of the European Union's Seventh Framework Programme FP7/20072013/ under REA grant agreement number PITN-GA-2011289313. We wish to extend thanks to those of Hawaiian ancestry on whose sacred mountain we are privileged guests.

Facilities: HST, Keck:II (DEIMOS)

\section{REFERENCES}

Athanassoula, E. 2002, ApJ, 569, L83

Athanassoula, E. 2003, MNRAS, 341, 1179

Athanassoula, E., \& Misiriotis, A. 2002, MNRAS, 330, 35

Athanassoula, E., \& Sellwood, J. A. 1986, MNRAS, 221, 213

Barnes, J. E., \& Hernquist, L. E. 1991, ApJ, 370, L65

Barton, E. J., Geller, M. J., Bromley, B. C., van Zee, L., \& Kenyon, S. J. 2001, AJ, 121,625

Bell, E. F., \& de Jong, R. S. 2001, ApJ, 550, 212

Cameron, E., Carollo, C. M., Oesch, P., et al. 2010, MNRAS, 409, 346

Capak, P. 2003, PhD thesis, Univ. Hawaii

Conselice, C. J., Bundy, K., Ellis, R. S., et al. 2005, ApJ, 628, 160

Courteau, S., Andersen, D. R., Bershady, M. A., MacArthur, L. A., \& Rix, H.-W. 2003, ApJ, 594, 208

Cowie, L. L., Songaila, A., Hu, E. M., \& Cohen, J. G. 1996, AJ, 112, 839

Cresci, G., Hicks, E. K. S., Genzel, R., et al. 2009, ApJ, 697, 115

Davies, R., Förster Schreiber, N. M., Cresci, G., et al. 2011, ApJ, 741, 69

Davis, M., Faber, S. M., Newman, J., et al. 2003, Proc. SPIE, 4834, 161 
Davis, M., Guhathakurta, P., Konidaris, N. P., et al. 2007, ApJ, 660, L1

Elmegreen, B. G., Elmegreen, D. M., Fernandez, M. X., \& Lemonias, J. J. 2009, ApJ, 692, 12

Elmegreen, D. M., Elmegreen, B. G., \& Hirst, A. C. 2004, ApJ, 604, L21

Elmegreen, D. M., Elmegreen, B. G., Ravindranath, S., \& Coe, D. A. 2007, ApJ, 658,763

Elmegreen, D. M., Elmegreen, B. G., Rubin, D. S., \& Schaffer, M. A. 2005, ApJ, 631,85

Fernández Lorenzo, M., Cepa, J., Bongiovanni, A., et al. 2009, A\&A, 496, 389

Förster Schreiber, N. M., Genzel, R., Bouché, N., et al. 2009, ApJ, 706, 1364

Gerin, M., Combes, F., \& Athanassoula, E. 1990, A\&A, 230, 37

Heller, C. H., Shlosman, I., \& Athanassoula, E. 2007, ApJ, 671, 226

Hohl, F. 1971, ApJ, 168, 343

Kalnajs, A. J. 1972, ApJ, 175, 63

Kannappan, S. J., Fabricant, D. G., \& Franx, M. 2002, AJ, 123, 2358
Kassin, S. A., Weiner, B. J., Faber, S. M., et al. 2007, ApJ, 660, L35

Kassin, S. A., Weiner, B. J., Faber, S. M., et al. 2012, arXiv:1207.7072

Law, D. R., Steidel, C. C., Erb, D. K., et al. 2009, ApJ, 697, 2057

Mihos, J. C., \& Hernquist, L. 1994, ApJ, 431, L9

Mihos, J. C., \& Hernquist, L. 1996, ApJ, 464, 641

Miller, S. H., Bundy, K., Sullivan, M., Ellis, R. S., \& Treu, T. 2011, ApJ, 741, 115

Newman, J. A., Cooper, M. C., Marc Davis, S. M., et al. 2012, ApJS, submitted (arXiv:1203.3192)

Ostriker, J. P., \& Peebles, P. J. E. 1973, ApJ, 186, 467

Romano-Díaz, E., Shlosman, I., Heller, C., \& Hoffman, Y. 2008, ApJ, 687, L13

Sellwood, J. A., \& Wilkinson, A. 1993, Rep. Prog. Phys., 56, 173

Sheth, K., Elmegreen, D. M., Elmegreen, B. G., et al. 2008, ApJ, 675, 1141

Weiner, B. J., Willmer, C. N. A., Faber, S. M., et al. 2006a, ApJ, 653, 1027

Weiner, B. J., Willmer, C. N. A., Faber, S. M., et al. 2006b, ApJ, 653, 1027

Wright, S. A., Larkin, J. E., Law, D. R., et al. 2009, ApJ, 699, 421 